\title{
Quantitative Analysis of the Bacteria in Blepharitis With Demodex Infestation
}

\author{
Minyi Zhu', Chao Cheng ${ }^{\dagger}$, Haisu Yi, Liping Lin and Kaili Wu*
}

Zhongshan Ophthalmic Center, State Key Laboratory of Ophthalmology, The Key Lab of Ophthalmology and Visual Science of Guangdong, Sun Yat-sen University, Guangzhou, China

Bacterial infection or Demodex infestation has been reported to contribute to chronic blepharitis. The association between Demodex mites and bacterial flora in this disease remains to be elucidated. Fifty-six consecutive patients diagnosed with chronic blepharitis and 46 healthy volunteers were recruited for this study. Using specimens of three epilated eyelashes and lid margin swabs, Demodex were identified microscopically

OPEN ACCESS

Edited by: George Grant, University of Aberdeen, United Kingdom

Reviewed by:

Heloisa D'Avila Da Silva Bizarro, Universidade Federal de Juiz de Fora,

Brazil

Daniel Ferreira Feijó,

Fiocruz Bahia, Brazil

*Correspondence:

Kaill Wu

wukaili@mail.sysu.edu.cn

tThese authors have contributed equally to this work.

Specialty section:

This article was submitted to Infectious Diseases,

a section of the journal

Frontiers in Microbiology

Received: 01 August 2017

Accepted: 10 July 2018

Published: 31 July 2018

Citation:

Zhu M, Cheng C, Yi H, Lin L and Wu K (2018) Quantitative Analysis of the Bacteria in Blepharitis With

Demodex Infestation.

Front. Microbiol. 9:1719.

doi: 10.3389/fmicb.2018.01719 and bacteria were determined by cultures, followed by colony counting and mass spectrometry. We found 191 Demodex mites, 161 D. folliculorum and $30 \mathrm{D}$. brevis, in 45 patients. Correspondingly, 101 Demodex, 63 D. folliculorum and 38 D. brevis, were found in 21 controls ( $p<0.05$, both). Bacterial culture-positivity was obtained in samples (eyelashes, lid margins, or both) from 54 patients and in eyelashes from 37 controls. The total colony counts and the incidences and colony counts of Propinibacterium acnes and Staphylococcus aureus from patients' eyelashes were significantly higher than that of the controls. Furthermore, bacterial colony counts in blepharitis patients' eyelashes with $D$. folliculorum were higher than that of controls with $D$. folliculorum $(p<0.01)$. Similarly, $P$. acnes colonies increased significantly in patients' eyelashes with $D$. folliculorum $(p<0.05)$. These results suggest that $D$. folliculorum and $P$. acnes have a role in the occurrence of the chronic blepharitis. Further studies are required to reveal the relationship between these two organisms in blepharitis.

Keywords: bacteria, demodex mites, blepharitis, eyelashes, MALDI-TOF-MS

\section{INTRODUCTION}

Blepharitis is a common eye condition characterized by inflammation of the eyelid margins, with a sensation of burning, stinging, and itching and clinical evidence of scales around the eyelashes, lid margin erythema, telangiectasia, thickening, irregularity of the eyelid margins and conjunctival hyperemia. Very little is known of the incidence or prevalence of this disorder. In one study, it was reported that blepharitis was found in $37 \%$ of patients in the ophthalmologist's clinical practice in the United States (Lemp and Nichols, 2009). Another study on 1148 consecutive patients with ocular discomfort or irritation reported anterior blepharitis in $12 \%$ and posterior blepharitis in 24\% (Venturino et al., 2003). Multiple diseases and conditions, such as allergies, rosacea, clogged oil glands and/or meibomian gland dysfunction, as well as bacteria, viral, and Demodex mite infections, were considered to be the causes of blepharitis (Jackson, 2008; Liu et al., 2010). 
Blepharitis caused by various bacterial infection has been receiving considerable clinical attention. The most common bacteria in chronic blepharitis include Staphylococci, Corynebacterium, and Propronibacterium species (Dougherty and McCulley, 1984; Teweldemedhin et al., 2017). In one study of the microbiota of blepharitis comparing 332 consecutive patients with chronic blepharitis to 160 control patients, Groden et al. (1991) reported that the most commonly isolated organisms from lids with blepharitis were Staphylococcus epidermidis (95.8\%), Propinibacterium acnes (92.8\%), Corynebacterium spp. (76.8\%), Acinetobacter spp. (11.4\%), and Staphylococcus aureus (10.5\%) based on cultivable approaches. Similarly, by analyzing the $16 \mathrm{~S}$ rRNA microbiome from lashes and tears of people with and without blepharitis, Lee et al. (2012) found that the microbiome mainly consisted of five genera (i.e., Propionibacterium, Staphylococcus, Streptophyta, Corynebacterium, and Enhydrobacter), but the differences between people with and without blepharitis, especially for the microbiome, were not statistically significant. Thus, antibiotics are widely used for the treatment of blepharitis in clinical practice (Pflugfelder et al., 2014).

Two types of Demodex mites, Demodex folliculorum (D. folliculorum), and Demodex brevis (D. brevis), were identified in humans and were reported to be associated with eye disorders, such as blepharitis, dry eye, conjunctivitis, and corneal lesions (Gao et al., 2007; Liang et al., 2014). The prevalence of mite infestation increases with age, and $\sim 58 \%$ of individuals with mites will have evidence of chronic blepharitis (Elston, 2010). Dadaci reported that direct microscopic examination revealed Demodex mites in $42.1 \%$ of the blepharitis group (Dadaci et al., 2015). In the eyelids, D. folliculorum are frequently found in hair follicles, while D. brevis has been thought to live in sebaceous glands (meibomian and Zeiss glands) (Liu et al., 2010). Therefore, D. folliculorum was supposed to cause chronic anterior blepharitis, while D. brevis has been considered to cause posterior blepharitis (Cheng et al., 2015). Meanwhile, there have been some studies that have suggested that metronidazole and tea tree oil are effective choices for the treatment of demodex infestation in blepharitis (Cheng et al., 2015; Akcinar et al., 2016). However, most people who have these obligate mites are healthy and there is no rigorous evidence demonstrating that Demodex mite infestation promotes bacterial infection in blepharitis (Clifford and Fulk, 1990; Bezza Benkaouha et al., 2015). Exploring the reason that mites contribute to blepharitis will be beneficial to therapy. Recently, people have been making significant efforts to explore the important relationship between the presence of Demodex and blepharitis (Kosik-Bogacka et al., 2012; Bhandari and Reddy, 2014; Cheng et al., 2015). The Demodex mite was supposed to act as a carrier of Bacillus oleronius that was found inside the parasite, which may function as a co-pathogen in the pathogenesis of blepharitis (Lacey et al., 2007; Szkaradkiewicz et al., 2012).

The purpose of the present study was to investigate the bacterial flora in eyelid margins and hair follicles infested with Demodex in chronic blepharitis as well as to quantify the mite and bacteria loads compared with normal individuals. To the best of our knowledge, there is no previous report of a quantitative analysis of the bacteria between the pulled eyelashes and lid margin in chronic blepharitis with Demodex infestation. In our opinion, the discovery of specific bacteria associated with demodex infestation in blepharitis may contribute to improved blepharitis treatment.

\section{MATERIALS AND METHODS}

\section{Patients}

All patients were referred to the Zhongshan Ophthalmic Center between May 2016 and June 2017 and had a primary diagnosis of chronic blepharitis. A total of 56 patients were consecutively included in the study. Meanwhile, 46 normal subjects without ocular or systemic disorders and have not used topical or systemic antibiotics within 2 weeks, matched by age and gender, were recruited for the control group. Study subjects were examined using a slit-lamp biomicroscope to screen for the presence of ocular signs of blepharitis. The diagnosis was based on symptoms and the ocular findings, as described by AAO Blepharitis: Preferred Practice Pattern Guideline 2013 ${ }^{1}$. This study was approved by the Human Research Ethics Committee of Zhongshan Ophthalmic Center, and all subjects provided written informed consent.

\section{Demodex Examination}

The sampling of eyelashes for Demodex examination was conducted as we previously reported with modification (Li et al., 2010; Liang et al., 2014). Briefly, the Demodexes in patients with a cylindrical sleeve, crusting, scurf or matting were chosen. Three eyelashes, isolated and taken from the upper lid of a patient or a healthy subject by sterile forceps, were placed on a pre-sterilized, disposable glass slide. A drop of sterile saline was mounted on the Demodexes. Under the microscope, a positive result involved the presence of at least one adult, larva, protonymph, or nymph stage of $\mathrm{D}$. folliculorum or $\mathrm{D}$. brevis. In parallel, the number and species of Demodex were counted. The process of Demodex examination was performed under sterile conditions. For reviewing the results, all eyelid conditions and findings on slides were photographed with cameras built into microscopes. After checking and recording the Demodex, the sample (eyelashes \pm mites in saline) on the slide was immediately inoculated onto plates using FLOQSwabs ${ }^{\text {TM }}$ (Copan Italia, Brescia, Italy). An ophthalmic surgeon (MY Zhu) collected eyelash specimens from all subjects.

\section{Bacterial Culture and Identification}

A complete microbiologic evaluation of cultures from the eyelid margins and eyelashes was performed. All protocols were conducted as we previously reported with modification (Wang et al., 2015). Briefly, by using a moistened Copan flocked swab with sterile saline, the lid margin samples were obtained by wiping twice along the upper lid margin from the angle to the punctum. Then, the swabs were seeded onto a culture plate (chocolate agar + polyViteX, bioMérieux, Shanghai, China). In

\footnotetext{
${ }^{1}$ www.aao.org/ppp
} 
TABLE 1 | Prevalence of two Demodex mites in blepharitis patients and controls.

\begin{tabular}{|c|c|c|c|c|}
\hline & \multicolumn{2}{|c|}{ Blepharitis patients $(n=56)(\%)$} & \multicolumn{2}{|c|}{ Controls $(n=46)(\%)$} \\
\hline & Positive subjects & Total demodex & Positive subjects & Total demodex \\
\hline Demodex & $45(80.36)^{*}$ & 191 & 21 (45.65) & 101 \\
\hline D. folliculorum & $43(76.79)^{*}$ & $161(84.29)^{*}$ & $19(41.30)$ & $63(62.38)$ \\
\hline D. brevis & 17 (30.36) & 30 (15.71) & 15 (32.61) & 38 (37.62) \\
\hline
\end{tabular}

$* P<0.01$ (patient vs. control).

addition, the residues on glass slides, which were used for the Demodex examination, were inoculated onto the plate by the Copan flocked swab. Both aerobes and anaerobes were routinely cultured separately at $35^{\circ}$ for up to 7 days. The grown colonies were macroscopically evaluated and recorded.

Identification of the bacterial colony was performed using MALDI-TOF-MS (VITEK MS, bioMérieux, France) according to what we reported previously (Song et al., 2017). Briefly, a single colony of isolates grown on a plate was selected, transferred to a target slide, and overlaid with CHCA matrix solution (bioMérieux). Each sample that passed the auto-quality control was achieved. E. coli ATCC 8739 served as a calibrated control in the acquisition. For multiple types of colonies on a plate, a representative colony from each type was separately identified by MALDI-TOF-MS and noted.

\section{Statistical Analysis}

A Chi-square test was used for frequency data analysis and the Mann-Whitney test for attributes data analysis. All statistics were performed with SPSS 20.0, and the level of significance for all tests was adopted as 0.05 .

\section{RESULTS}

\section{Demodex Findings}

A total of 56 patients ( 32 females and 24 males, $34.3 \pm 16.3$ years) and 46 normal individuals (29 females and 17 males, $40.4 \pm 19.2$ years), matched by age and gender, were consecutively recruited for this study. Demodex was found in $80.36 \%(45 / 56)$ of patients with blepharitis and $45.65 \%$ $(21 / 46)$ of controls $(p<0.01)$. The average number of Demodex mites on three eyelashes per patient was $3.41 \pm 3.86$, while the average was $2.20 \pm 3.66$ in control subjects $(p<0.01)$. There were 191 Demodex (D. folliculorum: 161, D. brevis: 30) found in blepharitis patients. Additionally, 101 Demodex (D. folliculorum: 63, D. brevis: 38 ) were found in controls. For the incidence and counts of D. folliculorum, the difference was significant between blepharitis patients and controls $(p<0.01)$, while no significant difference was observed for D. brevis ( $p>0.05$; Table 1).

\section{Bacteriological Findings}

Culture of patient samples revealed culture-positivity in 54 samples of eyelashes (96.55\%) and 54 samples of lid margins (96.55\%) from 56 blepharitis patients and in 37 of $46(80.43 \%)$ eyelashes from healthy individuals. There were 27 species of
16 genera of bacteria in blepharitis samples, while only 16 species of 11 genera, in healthy individuals. Table 2 displays major bacteria for three types of samples. Propionibacterium spp., Staphylococcus spp., and Bacillus spp. were the most frequently isolated organisms from all individuals. For anaerobic bacteria recovered from subjects, Propionibacterium spp., Bacillus spp., and Fusobacterium spp. were the most frequently isolated organisms. Other relatively infrequently isolated organisms included Streptococcus spp., Corynebacterium spp., Micrococcus spp., Neisseria spp., and others. In blepharitis patients, the prevalence and categories of isolates from the lid margin were similar to eyelashes. However, the bacterial distribution on eyelashes was different between the blepharitis patients and controls. There were significant differences in the culture-positive incidence of $P$. acnes and $S$. aureus between blepharitis patients and controls based on eyelash culture (Table 2).

We further quantified the isolates from different sample groups by counting the colonies. There were 2784 colonies (P. acnes, 1341; S. aureus, 331; and S. epidermidis, 335) on culture plates from the lid margins sand 2941 colonies ( $P$. acnes, 1113; S. aureus, 454; and S. epidermidis, 287) for eyelashes of 56 blepharitis patients, and 212 colonies ( $P$. acnes, 40; $S$ aureus, 27; and S. epidermidis, 59) for eyelash culture of 46 controls. Figure 1 demonstrates the colonies found in every subject for all three major bacteria in three sampling groups. The colony counts in eyelashes of blepharitis patients (52.52 colonies/person)

TABLE 2 | Culture-positive incidences of major isolates in blepharitis patients and controls.

\begin{tabular}{|c|c|c|c|}
\hline & \multicolumn{2}{|c|}{ Blepharitis patients $(n=56)(\%)$} & \multirow{2}{*}{$\begin{array}{l}\text { Control eyelashes } \\
\qquad(n=46)(\%)\end{array}$} \\
\hline & Lid margin & Eyelash & \\
\hline \multicolumn{4}{|l|}{ Aerobic } \\
\hline Staphylococcus spp. & $36(61.29)$ & 39 (69.64) & $27(58.70)$ \\
\hline S. aureus & 22 (39.29) & $27(48.21)^{*}$ & $12(26.09)$ \\
\hline S. epidermidis & 22 (39.29) & $26(46.43)$ & $21(45.65)$ \\
\hline Streptococcus spp. & $12(21.43)$ & $13(23.21)^{* *}$ & $2(4.35)$ \\
\hline Corynebacterium spp. & $8(14.29)$ & $12(21.43)$ & $4(8.70)$ \\
\hline \multicolumn{4}{|l|}{ Anaerobic } \\
\hline Propionibacterium spp. & $42(75.00)$ & $42(75.00)^{*}$ & $13(28.26)$ \\
\hline P. acnes & $41(73.21)$ & $42(75.00)^{*}$ & $13(28.26)$ \\
\hline Bacillus spp. & $11(19.64)$ & $12(21.43)$ & $5(10.87)$ \\
\hline Total organisms & $54(96.43)$ & $54(96.43)^{* *}$ & $37(80.43)$ \\
\hline
\end{tabular}

${ }^{*} p<0.05$ and $^{* *} p<0.01$ (blepharitis vs. Control). 

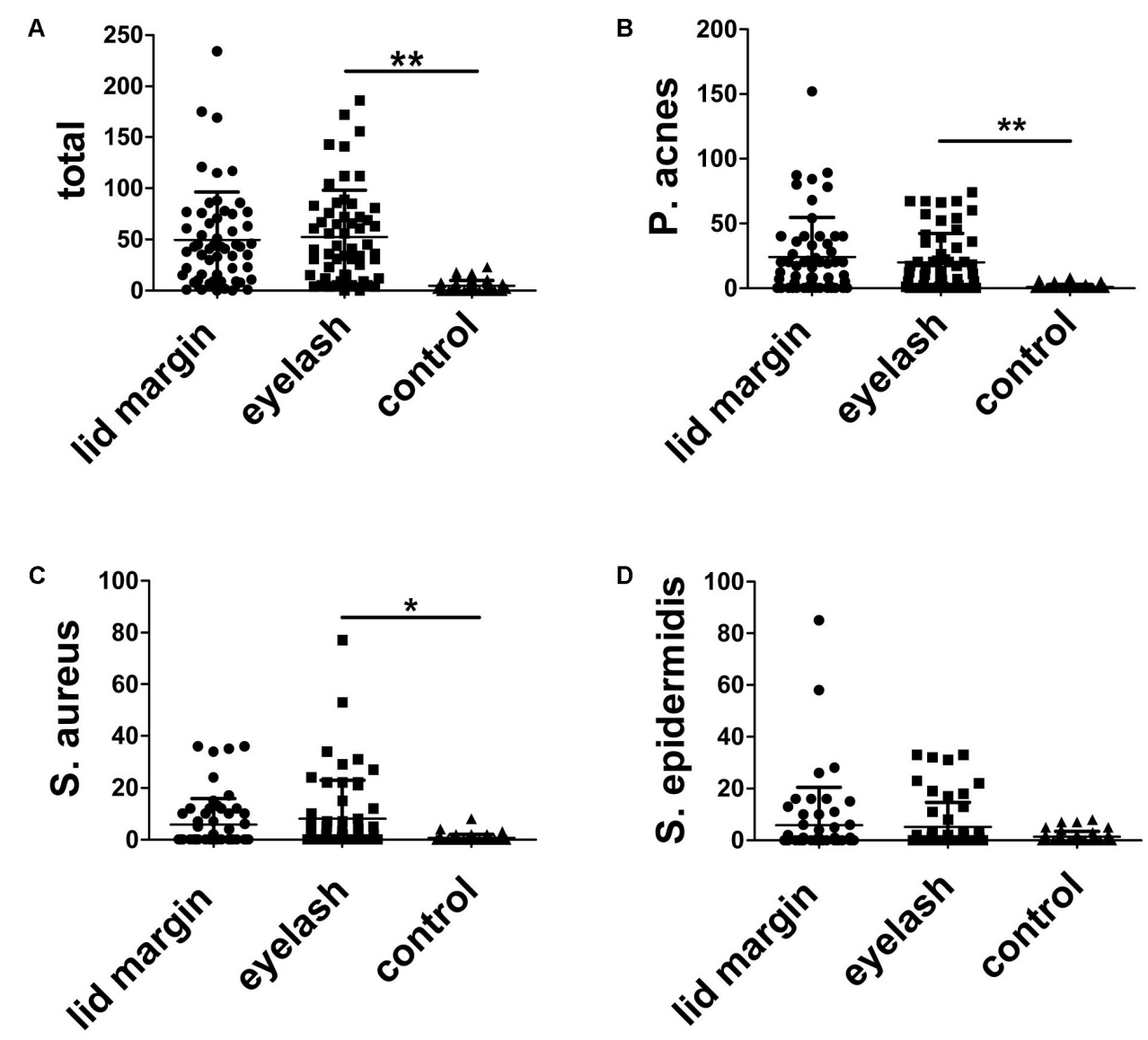

FIGURE 1 | Comparison of the colonies of the total and three major bacteria among different sample groups. The counted colonies of total bacterial isolated colonies (A), Propinibacterium acnes (B), and Staphylococcus aureus (C) from the eyelashes of patients were, separately, significantly more than that of normal individuals. (D) As for Staphylococcus epidermidis, no significant differences were observed among different groups. Lid margin, lid margin of blepharitis; eyelash, eyelash of blepharitis; control, eyelash of normal individuals. ${ }^{*} p<0.05$ and $* * p<0.01$.

were 10-fold higher than the control group (5.02 colonies/person) $(p<0.01)$. As expected, the colonies of both P. acnes and S. aureus were significantly higher in patient eyelashes than the normal group ( $p<0.05$, for both bacteria). However, the colony count of $S$. epidermidis in eyelashes did not show differences between patients and controls $(p>0.05)$. In addition, the bacterial loads of all three major isolates were the same between sampled eyelashes and lid margins in blepharitis patients $(p>0.05)$.

TABLE 3 | Incidences of major bacteria in the eyelashes of people with Demodex infestation.

\begin{tabular}{lcc}
\hline & $\begin{array}{c}\text { Eylash of patients } \\
\boldsymbol{n}=\mathbf{4 5}(\mathbf{\%})\end{array}$ & $\begin{array}{c}\text { Eyelash of control } \\
\boldsymbol{n}=\mathbf{2 1}(\%)\end{array}$ \\
\hline P. acnes & $38(84.44)^{*}$ & $12(57.14)$ \\
S. aureus & $18(40.00)$ & $7(33.33)$ \\
S. epidermidis & $23(51.11)$ & $10(57.62)$ \\
Others & $39(86.67)$ & $12(57.14)$ \\
Total & $45(100)^{*}$ & $17(80.95)$ \\
\hline
\end{tabular}

${ }^{*} p<0.05$ (blepharitis vs. control).

\section{Demodex Mites and Bacteria Coexisted in Sampled Eyelashes}

As described in Table 3, 45 patients had positive findings for Demodex mites and all of them had positive bacterial cultures. Correspondingly, 17 out of 21 healthy individuals with Demodex had positive cultures for bacteria based on eyelashes. Meanwhile, the prevalence of $P$. acne displayed significant increases in patient eyelashes with Demodex ( $p<0.05$, vs. control).

We further analyzed the relationship between the bacteria colonies and D. folliculorum infestation (Figure 2). The total bacterial loads in samples with $\mathrm{D}$. folliculorum from lid margins and eyelashes of patients and eyelashes of control were 2400, 2521, and 116 colonies, respectively. For samples with D. folliculorum infestation, there were significant increases in the bacterial colonies in patients eyelashes compared to healthy people $(p<0.01)$. Similarly, the colonies of $P$. acnes increased significantly in samples with $\mathrm{D}$. folliculorum infestation from patients as well as healthy individuals $(p<0.05)$. The loads of the other two bacteria, i.e., S. aureus and S. epidermidis, did not show differences if there was a $D$. folliculorum infestation. 

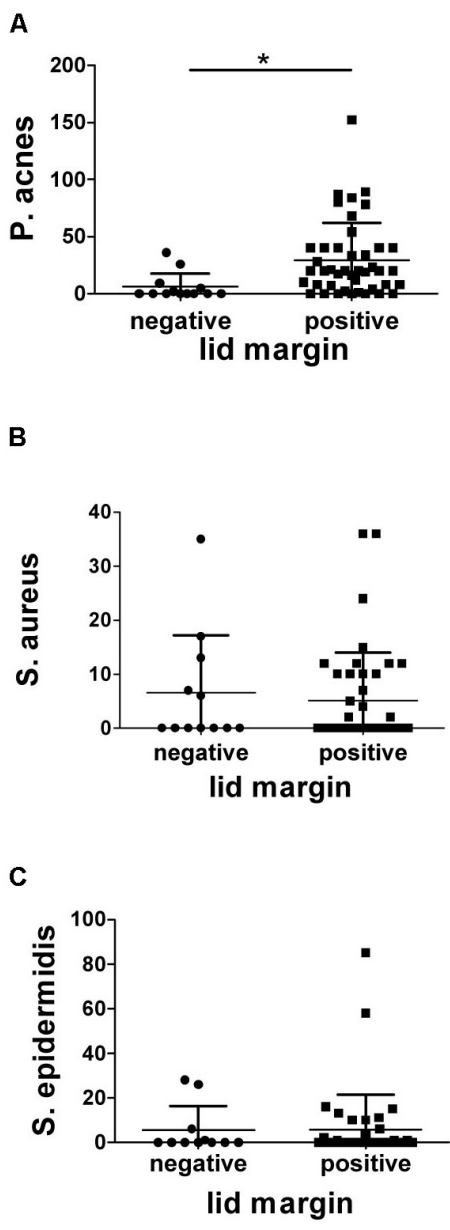
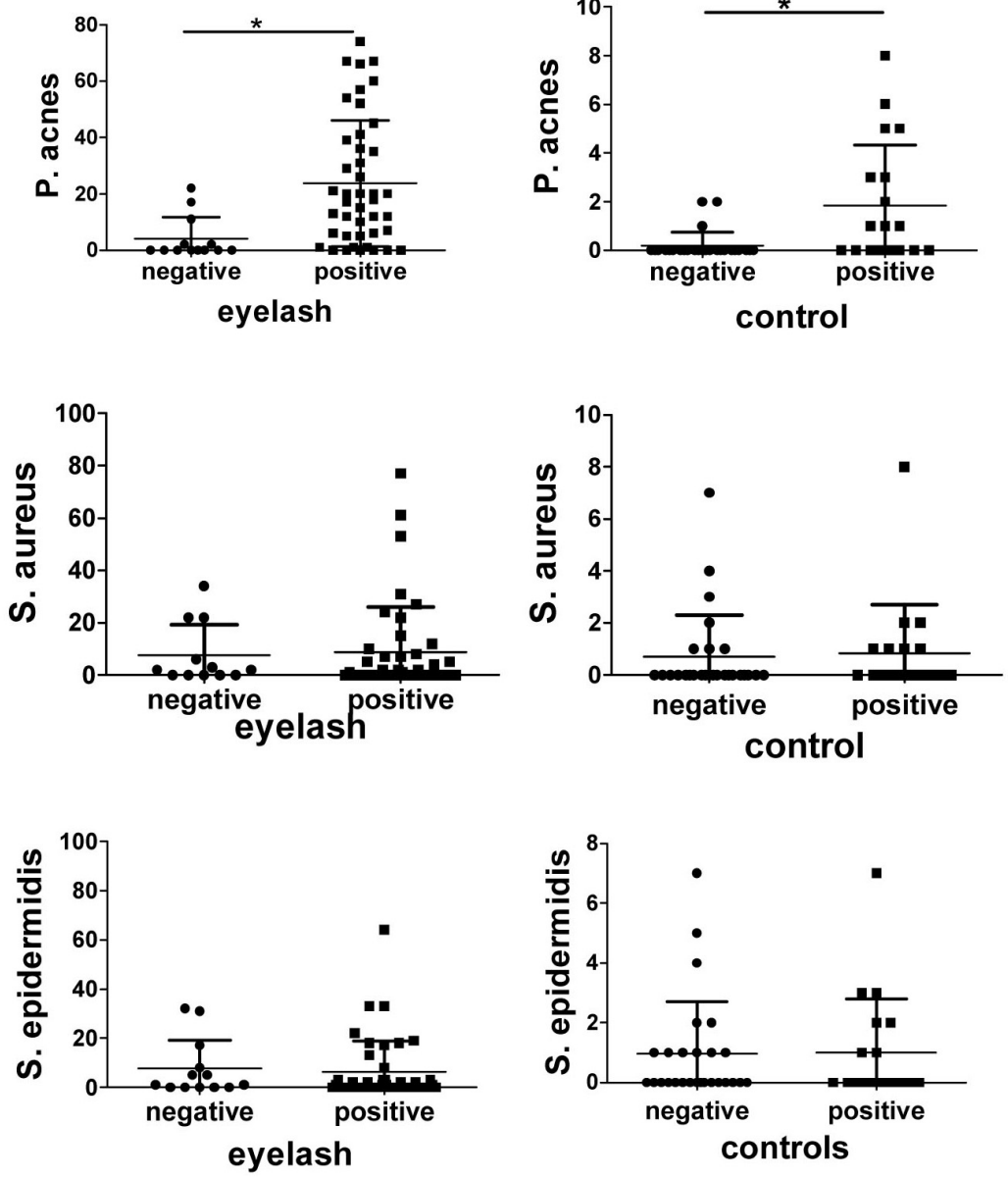
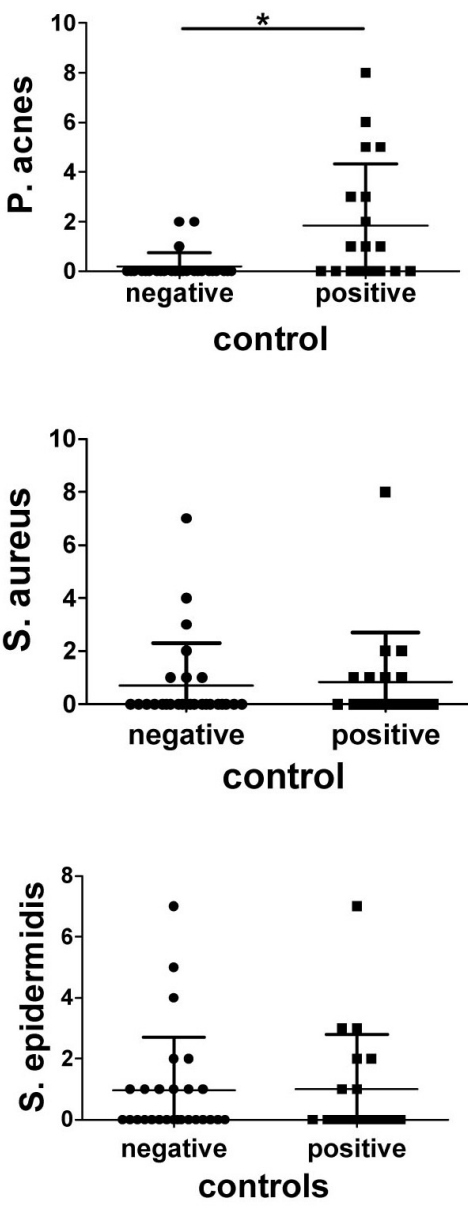

FIGURE 2 | Comparisons of bacterial colonies in samples with or without D. folliculorum infestation. (A) Shows that the P. acnes colonies were significantly increased in samples with positive D. folliculorum $(p<0.05)$. By contrast, the colonies of $S$. aureus (B) and $S$. epidermidis (C) did not have differences in the presence or absence of D. folliculorum infestation in the three types of culture samples. Lid margin, lid margin of blepharitis; eyelash, eyelash of blepharitis; control, eyelash of normal individuals. ${ }^{*} p<0.05$.

\section{DISCUSSION}

Blepharitis can arise from the infestation of Demodex mites and/or bacterial infection. In this study, we compared the bacterial flora on eyelashes with or without Demodex mites and on the lid margin in chronic blepharitis patients. We found that there was a higher prevalence of Demodex mites and bacteria, and that there were increased colony counts in the eyelash follicles in blepharitis compared to normal subjects. More importantly, the prevalence of bacteria culture revealed a significant increase in eyelashes with Demodex infestation. The colony count of $P$. acnes in samples that were positive for D. folliculorum was significantly higher than those without D. folliculorum in all three samples from lid margins and eyelashes of patients as well as eyelashes from control individuals. In addition, the bacterial loads and categories of the lid margin were similar to those from eyelashes.

In the present study, cultures of material obtained from the three types of samples yielded similar organisms at different frequencies. The percentages of the positive cultures and bacterial loads were significantly higher for blepharitis patients compared with normal controls, which is consistent with previous studies (Willcox, 2013; Bezza Benkaouha et al., 2015). Those studies showed that the bacteria frequently found on the eyelid margin of chronic blepharitis, including Staphylococcus, Propronibacterium, and Corynebacterium species, were also commonly isolated from healthy eyelids. Meanwhile, early studies emphasized that S. aureus was the predominant isolate in blepharitis with approximately half of patients compared to $<20 \%$ in normal individuals being positive cultures for $S$. aureus (Dougherty and McCulley, 1984; Jackson, 2008). Our data showed that two bacteria (i.e., S. aureus and P. acnes) displayed significantly higher positive rates on the eyelashes of patients compared to that of controls (Figure 1 and Table 2). Similarly, in our previous studies on the bacterial spectra and antibiotic resistance patterns of ocular infections, we found that the most prevalent bacterial genera was Staphylococcus spp., of which, S. epidermidis accounts for $35.25 \%$ of the external ocular infection and $14.96 \%$ of the intraocular infection (Wang et al., 2015, 2016). However, 
S. epidermidis, which was nearly the most frequently isolated bacterium with the highest loads on eyelid margins of normal subjects as well as blepharitis patients, was also reported to have a significantly increased frequency in the chronic blepharitis patients at different stages (Groden et al., 1991) or those exposed to mustard gas (Karimian et al., 2011). Our present study revealed that there were no significant differences for the positive rates of $S$. epidermidis isolated among the eyelid margins of patients and eyelashes of patients and healthy subjects. These results may suggest that the bacteria, excluding S. epidermidis, contributed to the pathogenesis in our patients.

In addition, the incidence of Streptococci spp. was significantly higher in blepharitis patients as compared to that in healthy subjects (13/56 vs. 2/46, Table 2). Because of the small number of positive cultures, we only presented the total number of Streptococci spp. and did not analyze the data for different species of Streptococci. Furthermore, the loads of Streptococci spp. didn't increase significantly in blepharitis patients (data not shown). Thus, we can't assume that any species of streptococci is pathogenic in the present study.

The correlation between Demodex infestation of the eyelids and blepharitis has been investigated epidemiologically and clinically for decades. We present that there is a clearly significant increase in the mite infestation in the eyelashes of chronic blepharitis compared to healthy subjects. In addition, we found that $80.36 \%$ of blepharitis patients (vs. $45.65 \%$ of control) had Demodex mites. Similar results, i.e., a significantly high incidence and density of Demodex infestation in patients, were reported by different researchers (Zhao et al., 2012; Bhandari and Reddy, 2014). These data provided indirect support for mite-related blepharitis. Baima and Sticherling (2002) have proposed the pathological changes in the course of demodicosis before. The underlying mechanism remains to be further explored. On the other hand, there are several routine treatment options for patients with blepharitis, from maintaining eyelid hygiene to the use of antibiotics (Pflugfelder et al., 2014). Broadspectrum antibiotics alone are widely used for the treatment of bacteria infection in blepharitis patients even when a significant demodex infestation is present. Our current results challenge these approaches. The anti-demodex therapy was emphasized by some previous studies (Cheng et al., 2015; Akcinar et al., 2016). Therefore, apart from the application of antibacterial agents, a combination of anti-demodex therapy to reduce demodex count should be considered.

Additionally, the Demodex mite was considered to be the vector for bacteria, which was demonstrated by the finding of Bacillus oleronius inside Demodex mites as well as the positive serum immune-reactivity to bacillus proteins in patients with ocular Demodex infestation, facial rosacea, and blepharitis ( $\mathrm{Li}$ et al., 2010). Comparisons of our bacterial data of sampling eyelashes with or without Demodex in normal eyes and those diagnosed with blepharitis have identified new findings in the present study. We provided additional evidence to support this speculation, e.g., a high prevalence of Demodex mite infestation accompanied by higher incidences and loads of bacteria in the eyelashes as well as eyelid margin of patients with chronic blepharitis compared to healthy controls. Furthermore, we found that $P$. acnes colonies significantly increased in eyelashes with positive D. folliculorum. Bezza Benkaouha et al. (2015) conducted a similar study in a small number of subjects and did not find a significant difference for Demodex in 9 blepharitis patients $(2 / 9,22.2 \%)$ vs. 7 normal individuals $(2 / 7,28.6 \%)$. Our study further demonstrated a strong correlation between Demodex infestation, bacterial infection and chronic blepharitis.

Because Demodex is the most common obligate ectoparasite in humans, with $80 \%$ present on skin (Vollmer, 1996) and $45.65 \%$ on eyelashes (Table 1) in healthy people, a chronic infestation of mites in the eyelids may result in inflammation of the eyelid and ocular surface as well as secondary bacterial infection. The present study was a preliminary study that demonstrated synergic infection of bacteria and Demodex mites around the eyelashes in chronic blepharitis, but it has limitations that should be addressed. We only recruited patients with mixed blepharitis, which is usually more severe than any single type (e.g., anterior or posterior blepharitis) of this disorder, and it usually involves serious disruption of the biofilm on the eyelids (Rynerson and Perry, 2016). Accompanying Demodex infestation, the contribution of bacterial infection is more intensive in this condition. Also, it is likely that during infection/inflammation of the eyelid, the microflora of the eyelash will be "contaminated" by that of the eyelid through "pus" and other semi-liquid substances smearing the eyelashes, which will result in mixing of the microbial flora. Any approach that can avoid this kind of contamination will be valuable for the future study. In addition, the low sample size has prevented deep analyses on the subtype of bacteria and Demodex mites. These will be overcome by recruiting more patients at various statuses and by combining analyses on the molecular level in our future work.

According to this study, we conclude that in blepharitis patients, the bacterial loads sampled from eyelashes and lid margins were similar, which were abnormally different from healthy volunteers. There was a higher prevalence of Demodex mites in blepharitis patients compared to normal subjects. Our results suggested a correlation between D. folliculorum and $P$. acnes in blepharitis. Further studies are required to reveal the relationship between these two organisms in blepharitis.

\section{ETHICS STATEMENT}

This study was carried out in accordance with the recommendations of the "Regulations for the Human Research from the Ethics Committee of Zhongshan Ophthalmic Center" with written informed consent from all subjects. All subjects gave written informed consent in accordance with the Declaration of Helsinki. The protocol was approved by the "Human Research Ethics Committee of Zhongshan Ophthalmic Center."

\section{AUTHOR CONTRIBUTIONS}

MZ identified patients, collected eye specimens, observed demodex mites, cultured bacteria, took photos, and drafted 
the manuscript. CC cultured, quantified, identified bacteria, and analyzed data. HY handled mass spectrometer and helped CC use mass spectrometer. LL organized control subjects, collected patients, and performed demodex analysis. KW supervised the study, participated in the discussions of each section of experiments, supported this research and reviewed manuscript. All authors read and approved the final manuscript.

\section{REFERENCES}

Akcinar, U. G., Unal, E., and Akpinar, M. (2016). Demodex spp. infestation associated with treatment-resistant Chalazia and Folliculitis. Turkiye Parazitol. Derg. 40, 208-210. doi: 10.5152/tpd.2016.4869

Baima, B., and Sticherling, M. (2002). Demodicidosis revisited. Acta Derm. Venereol. 82, 3-6. doi: 10.1080/000155502753600795

Bezza Benkaouha, I., Le Brun, C., Pisella, P. J., Chandenier, J., and Lanotte, P. (2015). Bacterial flora in blepharitis. J. Fr. Ophtalmol. 38, 723-728. doi: 10.1016/ j.jfo.2015.01.012

Bhandari, V., and Reddy, J. K. (2014). Blepharitis: always remember Demodex. Middle East Afr. J. Ophthalmol. 21, 317-320. doi: 10.4103/0974-9233.142268

Cheng, A. M., Sheha, H., and Tseng, S. C. (2015). Recent advances on ocular Demodex infestation. Curr. Opin. Ophthalmol. 26, 295-300. doi: 10.1097/ICU. 0000000000000168

Clifford, C. W., and Fulk, G. W. (1990). Association of diabetes, lash loss, and Staphylococcus aureus with infestation of eyelids by Demodex folliculorum (Acari: Demodicidae). J. Med. Entomol. 27, 467-470. doi: 10.1093/jmedent/27. 4.467

Dadaci, Z., Kilinc, F., Ozer, T. T., Sahin, G. O., Acir, N. O., and Borazan, M. (2015). Periodic acid-Schiff staining demonstrates fungi in chronic anterior blepharitis. Eye 29, 1522-1527. doi: 10.1038/eye.2015.144

Dougherty, J. M., and McCulley, J. P. (1984). Comparative bacteriology of chronic blepharitis. Br. J. Ophthalmol. 68, 524-528. doi: 10.1136/bjo.68.8.524

Elston, D. M. (2010). Demodex mites: facts and controversies. Clin. Dermatol. 28, 502-504. doi: 10.1016/j.clindermatol.2010.03.006

Gao, Y. Y., Di Pascuale, M. A., Elizondo, A., and Tseng, S. C. (2007). Clinical treatment of ocular demodecosis by lid scrub with tea tree oil. Cornea 26, 136-143. doi: 10.1097/01.ico.0000244870.62384.79

Groden, L. R., Murphy, B., Rodnite, J., and Genvert, G. I. (1991). Lid flora in blepharitis. Cornea 10, 50-53. doi: 10.1097/00003226-199110010-00010

Jackson, W. B. (2008). Blepharitis: current strategies for diagnosis and management. Can. J. Ophthalmol. 43, 170-179. doi: 10.1139/i08-016

Karimian, F., Zarei-Ghanavati, S., A, B. R., Jadidi, K., and Lotfi-Kian, A. (2011). Microbiological evaluation of chronic blepharitis among Iranian veterans exposed to mustard gas: a case-controlled study. Cornea 30, 620-623. doi: 10.1097/ICO.0b013e3181e16f7c

Kosik-Bogacka, D. I., Lanocha, N., Lanocha, A., Czepita, D., Grobelny, A., Zdziarska, B., et al. (2012). Role of Demodex folliculorum in the pathogenesis of blepharitis. Acta Ophthalmol. 90:e579. doi: 10.1111/j.1755-3768.2012.02391.x

Lacey, N., Delaney, S., Kavanagh, K., and Powell, F. C. (2007). Mite-related bacterial antigens stimulate inflammatory cells in rosacea. Br. J. Dermatol. 157, 474-481. doi: 10.1111/j.1365-2133.2007.08028.x

Lee, S. H., Oh, D. H., Jung, J. Y., Kim, J. C., and Jeon, C. O. (2012). Comparative ocular microbial communities in humans with and without blepharitis. Invest. Ophthalmol. Vis. Sci. 53, 5585-5593. doi: 10.1167/iovs.12-9922

Lemp, M. A., and Nichols, K. K. (2009). Blepharitis in the United States 2009: a survey-based perspective on prevalence and treatment. Ocul. Surf. 7(2 Suppl.), S1-S14. doi: 10.1016/S1542-0124(12)70620-1

Li, J., O’Reilly, N., Sheha, H., Katz, R., Raju, V. K., Kavanagh, K., et al. (2010). Correlation between ocular Demodex infestation and serum immunoreactivity to Bacillus proteins in patients with Facial rosacea. Ophthalmology 117, 870.e1877.e1. doi: 10.1016/j.ophtha.2009.09.057

\section{FUNDING}

This work was partially supported by grants the Guangzhou Science Technology and Innovation Commission (201607020011), the Natural Science Foundation of Guangdong (2014A030313162), and the Science and Technology Planning Project of Guangdong Province (2014B030301040), China.

Liang, L., Ding, X., and Tseng, S. C. (2014). High prevalence of demodex brevis infestation in chalazia. Am. J. Ophthalmol. 157, 342.e1-348.e1. doi: 10.1016/j. ajo.2013.09.031

Liu, J., Sheha, H., and Tseng, S. C. (2010). Pathogenic role of Demodex mites in blepharitis. Curr. Opin. Allergy Clin. Immunol. 10, 505-510. doi: 10.1097/ACI. 0b013e32833df9f4

Pflugfelder, S. C., Karpecki, P. M., and Perez, V. L. (2014). Treatment of blepharitis: recent clinical trials. Ocul. Surf. 12, 273-284. doi: 10.1016/j.jtos.2014. 05.005

Rynerson, J. M., and Perry, H. D. (2016). DEBS - a unification theory for dry eye and blepharitis. Clin. Ophthalmol. 10, 2455-2467. doi: 10.2147/OPTH.S11 4674

Song, H., Liu, X., Zhu, M., Tan, Y., and Wu, K. (2017). Using MALDI-TOF-MS to test Staphylococcus aureus-infected vitreous. Mol. Vis. 23, 407-415.

Szkaradkiewicz, A., Chudzicka-Strugala, I., Karpinski, T. M., GoslinskaPawlowska, O., Tulecka, T., Chudzicki, W., et al. (2012). Bacillus oleronius and Demodex mite infestation in patients with chronic blepharitis. Clin. Microbiol. Infect. 18, 1020-1025. doi: 10.1111/j.1469-0691.2011.03704.x

Teweldemedhin, M., Saravanan, M., Gebreyesus, A., and Gebreegziabiher, D. (2017). Ocular bacterial infections at Quiha Ophthalmic Hospital, Northern Ethiopia: an evaluation according to the risk factors and the antimicrobial susceptibility of bacterial isolates. BMC Infect. Dis. 17:207. doi: 10.1186/s12879017-2304- 1

Venturino, G., Bricola, G., Bagnis, A., and Traverso, C. (2003). Chronic blepharitis: treatment patterns and prevalence. 2003;44:E-Abstract 774. Invest. Ophthalmol. Vis. Sci. 44:e774.

Vollmer, R. T. (1996). Demodex-associated folliculitis. Am. J. Dermatopathol. 18, 589-591. doi: 10.1097/00000372-199612000-00006

Wang, N., Huang, Q., Tan, Y. W., Lin, L. P., and Wu, K. L. (2016). Bacterial spectrum and resistance patterns in corneal infections at a Tertiary Eye Care Center in South China. Int. J. Ophthalmol. 9, 384-389. doi: 10.18240/ijo.2016. 03.10

Wang, N., Yang, Q., Tan, Y., Lin, L., Huang, Q., and Wu, K. (2015). Bacterial spectrum and antibiotic resistance patterns of ocular infection: differences between external and intraocular diseases. J. Ophthalmol. 2015:813979. doi: $10.1155 / 2015 / 813979$

Willcox, M. D. (2013). Characterization of the normal microbiota of the ocular surface. Exp. Eye Res. 117, 99-105. doi: 10.1016/j.exer.2013.06.003

Zhao, Y. E., Wu, L. P., Hu, L., and Xu, J. R. (2012). Association of blepharitis with Demodex: a meta-analysis. Ophthalmic Epidemiol. 19, 95-102. doi: 10.3109/ 09286586.2011 .642052

Conflict of Interest Statement: The authors declare that the research was conducted in the absence of any commercial or financial relationships that could be construed as a potential conflict of interest.

Copyright (c) $2018 \mathrm{Zhu}$, Cheng, Yi, Lin and Wu. This is an open-access article distributed under the terms of the Creative Commons Attribution License (CC BY). The use, distribution or reproduction in other forums is permitted, provided the original author(s) and the copyright owner(s) are credited and that the original publication in this journal is cited, in accordance with accepted academic practice. No use, distribution or reproduction is permitted which does not comply with these terms. 